\title{
Note: Electrical modeling and characterization of voltage gradient in liquid crystal microlenses
}

\author{
V. Urruchi, ${ }^{\text {a) }}$ J. F. Algorri, C. Marcos, and J. M. Sánchez-Pena \\ Departamento de Tecnología Electrónica, Universidad Carlos III, Butarque 15, \\ E28911 Leganés, Madrid, Spain
}

(Received 2 October 2013; accepted 6 November 2013; published online 18 November 2013)

\begin{abstract}
In this work, a novel equivalent electric circuit for modeling liquid crystal microlenses is proposed. This model is focused on explaining a lens behavior at the micrometric scale, using its manufacturing parameters. It suggests an approach to predict the solution of the voltage gradient distribution across a microlens. An interesting feature of the model is that it provides an analytical solution for microlenses with modal and hole-patterned electrode schemes, by a simple software tool. The model flexibility allows lens designers to apply complex waveform signals with different harmonics. The voltage distribution has been tested. The simulated and measured voltage profiles are fairly in agreement. (C) 2013 AIP Publishing LLC. [http://dx.doi.org/10.1063/1.4832419]
\end{abstract}

The design of new liquid crystal (LC) microlenses is involving currently important research resources. Prototypes based on this technology take advantage of the tunable electrooptical properties of these materials mainly for the focal length tunability. This distinctive feature together with a reduced weight and volume of the devices, compared to other technologies, makes them good candidates for a wide range of applications. Cell phone cameras, auto-focusing, 2D/3D switchable displays, tunable photonic devices, or devices in optical communication systems are potential applications for providing final added value. In order to simplify the structures and to decrease the device control voltage, many topologies have been researched. Furthermore, the capacity of manufacturing LC microlenses is limited at the micrometric scale with the constraint of lithographic resolution. Some approaches, such as Fresnel zone method, with arrangements of multiple electrodes, complicate the final design. Other strategies consist of creating a lens effect by shaping the electric field generated with a hole-patterned electrode structure. In holepatterned electrode structures, the phase gradient is generated by the fringe field as the diameter/thickness ratio is less than 2-3. ${ }^{1}$ Fig. 1(a) shows the design of a cylindrical microlens following this approach; a top view of its hole-patterned electrode is depicted in the upper part. The spreading of the electric field lines, yielded from the teeth of the comb electrode to the center, creates a gradient of the LC director profile that simulates a lens response. Nevertheless, this approach does not work properly as the lens aperture increases in size, because of the electric field vanishes gradually without reaching the entire area of the hole. An option to mitigate this problem is to exploit the modal control technique. This method consists in adding a layer of high sheet resistance in areas which are free of the comb electrode. Fig. 1(b) illustrates a modal cylindrical microlens; in this arrangement, an electric field gradient is generated across the high resistivity layer.

a) Author to whom correspondence should be addressed. Electronic mail: vurruchi@ing.uc3m.es.
This inclusion can be a possible solution to avoid the use of several electrodes in lenses with wider apertures. On the contrary, modal design becomes a handicap as size gets smaller ${ }^{2}$ and involves a very careful design of the control layer resistivity. That is, the smaller the lens size, the higher the sheet resistance of the layer to keep an equivalent field distribution on the lens surface for lenses with different sizes. Besides, we have evidenced for the first time, to the best of our knowledge, that at the micrometric scale, the modal control layer is needless as the fringe field effect is predominant in a coplanar electrode structure.

The electric field distribution has already been modeled for modal lenses by a capacitive-resistive voltage divider in the aperture area. ${ }^{3}$ In this work, we describe a novel equivalent electrical circuit (EEC) based on a transmission line, which models also lenses with a hole-patterned electrode structure and establishes the hypothesis for implementing both topologies. The model determines a powerful tool to predict both modal and hole-patterned electrical responses during the design process. It involves an analytic study simpler than the complex finite element analysis. The new EEC consists of two kinds of components in the direction of the wave propagation: resistors, $\mathrm{R}$, and singular coplanar capacitors, $\mathrm{C}_{1}$ (Fig. 2). The LC is modeled with transverse components, perpendicular to the direction of the wave propagation: distributed capacitors, $\mathrm{C}_{2}$, in parallel with distributed conductances, G, per unit area, respectively. The last two components make the model only valid in a certain range of frequencies (100 Hz to $10 \mathrm{kHz}){ }^{4}$

In a modal topology, distributed resistors are $\mathrm{R}=\mathrm{R}_{\mathrm{sq}}$, that is, the sheet resistance connected to model the control layer. Alternatively, in a hole-patterned structure, distributed resistors connected in the same place, $\mathrm{R}=\mathrm{R}_{\mathrm{CL}}$, model the $\mathrm{LC}$ dielectric losses in the lens surface.

Distributed capacitors, $\mathrm{C}_{1}$, describe the capacitive coupling between fingers of the comb geometry electrode of the cylindrical lens that is caused by the fringe field. This capacitor results from the LC permittivity in the horizontal direction. Research in the field of coplanar capacitances has 


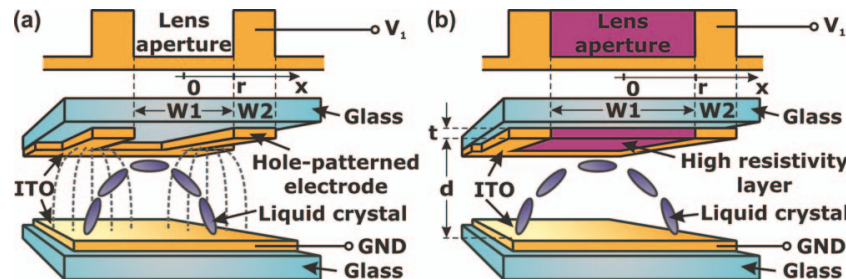

FIG. 1. (a) The hole-patterned electrode structure and (b) the modal control technique for a LC cylindrical microlens. Note that, devices have not been drawn to scale and, for clarity of the graph, the alignment layers have not been outlined.

given rise to a lot of works related to multiple applications: MOSFET transistors, ${ }^{5}$ microstrip lines, ${ }^{6}$ etc. One of the most accepted techniques for obtaining these capacitors is the theory of conformal transformations. Conformal mapping preserves the angles, transforming the structures to a complex plane. The result regions are simpler and easier to study. ${ }^{7}$ For analytical purposes in these settings, an approximation to the problem has been taken from the coplanar capacitors theory. ${ }^{8}$ The result is an equivalent parasitic capacitor, $\mathrm{C}_{1}$, inversely proportional to a constant A described in Eq. (1). Constant A has been estimated using conformal mapping based on the Christoffel-Schwarz transformation,

$$
A=\frac{2 K(k)}{K\left(k^{\prime}\right)} \quad \text { with } \quad k=\frac{W_{1}}{W_{1}+2 W_{2}}, \quad k^{\prime}=\sqrt{1-k^{2}},
$$

where $\mathrm{W}_{1}$ is the space between electrodes (the cylindrical lens diameter), $\mathrm{W}_{2}$ the electrode width, $\mathrm{K}(\mathrm{k})$ the complete elliptic integral of the first kind, and k the elliptic modulus. Finally, the components of the model are determined by,

$$
\begin{aligned}
R & =\frac{\rho}{t}\left(\frac{\Omega}{s q}\right) ; \quad C_{1}=\frac{\varepsilon_{0} \cdot \varepsilon_{1}^{\prime} \cdot d}{A}(F), \\
C_{2} & =\frac{\varepsilon_{0} \cdot \varepsilon_{2}^{\prime}}{d}\left(\frac{F}{m^{2}}\right) ; \quad G=\frac{\sigma}{d}\left(\frac{S}{m^{2}}\right),
\end{aligned}
$$

where $\rho$ and $\mathrm{t}$ are the resistivity and the thickness of the control layer (in modal control), respectively, $\varepsilon_{0}$ the vacuum permittivity, d the LC thickness, $\sigma$ the LC conductivity, and $\varepsilon^{\prime}{ }_{1}$ and $\varepsilon_{2}^{\prime}$ the average real effective permittivities in the horizontal and vertical directions, respectively (for simplifying the analysis, average values have been considered). In addition, all of the EEC components are defined per unit length, $\Delta \mathrm{x}$ ( $x$-axis is the direction of the transmission line), for a proper estimation of the analytical expressions,

$$
R \cdot \Delta x\left(\frac{\Omega}{m}\right) ; \frac{C_{1}}{\Delta x}\left(\frac{F}{m}\right) ; C_{2} \cdot \Delta x\left(\frac{F}{m}\right) ; G \cdot \Delta x\left(\frac{S}{m}\right) .
$$

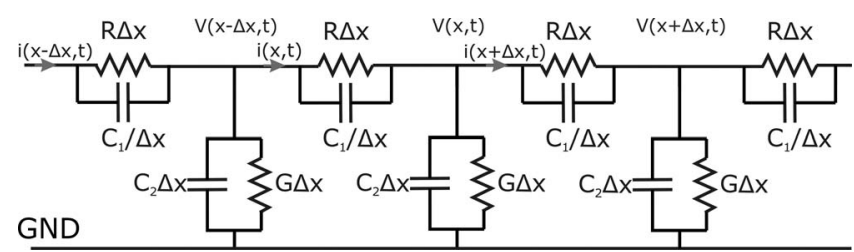

FIG. 2. Equivalent electrical circuit (EEC) for liquid crystal microlenses with coplanar capacitances $\mathrm{C}_{1}$.
Following the Ohm and Kirchhoff's laws, the current and voltage relations in the transmission line are,

$$
\begin{aligned}
i(x+\Delta x, t)= & \frac{V(x, t)-V(x+\Delta x, t)}{R \Delta x} \\
& +\frac{\partial V(x, t)-\partial V(x+\Delta x, t)}{\partial t \Delta x} C_{1},
\end{aligned}
$$

$$
i(x, t)-i(x+\Delta x, t)=C_{2} \Delta x \frac{\partial V(x, t)}{\partial t}+G \Delta x \cdot V(x, t),
$$

and, in order to estimate the voltage infinitesimal variation across a lens diameter, Eq. (4) is divided by $\Delta \mathrm{x}$ and the limit when $\Delta x$ tends to zero is calculated. The resulting system of equations, considering only one harmonic $V(x, t)$ $=\mathrm{V}(\mathrm{x}) \cdot \mathrm{e}^{\mathrm{j} \omega \mathrm{t}}$, is solved as a partial differential equation, Eq. (5),

$$
\frac{\partial V^{2}(x)}{\partial x^{2}}=\frac{G+j \omega C_{2}}{\frac{1}{R}+j \omega C_{1}} \cdot V(x)=\Psi \cdot V(x) .
$$

The solution to this ordinary differential equation depends on the boundary conditions, that is, the voltages at the two lens edges, $\mathrm{V}(\mathrm{x}=-\mathrm{r})$ and $\mathrm{V}(\mathrm{x}=\mathrm{r})$, with $\mathrm{x}$ the position on the lens aperture and $r$ the lens radius. Equation (6) gives the solution to the voltage distribution for two sets of boundary conditions,

$$
\begin{gathered}
\text { Case 1 }: V(-r)=V(r)=V_{1} \cdot e^{j \omega t} \\
\Rightarrow V(x)=V_{1} \frac{\cosh (\sqrt{\Psi} \cdot x)}{\cosh (\sqrt{\Psi} \cdot r)}, \\
\text { Case 2: }\left\{\begin{array}{l}
V(-r)=V_{1} \cdot e^{j \omega t} \\
V(r)=V_{1} \cdot e^{j \omega t+\pi}
\end{array}\right\} \\
\Rightarrow V(x)=V_{1} \frac{\sinh (\sqrt{\Psi} \cdot x)}{\sinh (\sqrt{\Psi} \cdot r)} .
\end{gathered}
$$

In addition to the previous calculations, voltage distribution has been necessarily obtained through an iterative process, for achieving an accurate result. Equation (7) connects the effective permittivities, $\varepsilon^{\prime}{ }_{1}$ and $\varepsilon^{\prime}{ }_{2}$, the dielectric anisotropy $\Delta \varepsilon$ (voltage dependent), and a new parameter $\mathrm{p}$ proportional to the average tilt angle of molecules,

$$
\frac{\varepsilon_{1}^{\prime}}{\varepsilon_{2}^{\prime}}=\frac{\Delta \varepsilon \cdot p+\varepsilon_{o}}{\Delta \varepsilon \cdot(1-p)+\varepsilon_{o}} \text { with } 0<p<1 .
$$

The iterative process for obtaining $\mathrm{V}(\mathrm{x})$ follows the program steps:

(i) First, LC molecules are considered in a relaxed position $(\mathrm{p}=1)$ for homogeneous alignment. Then, by Eq. (7), $\varepsilon^{\prime}{ }_{1}=\varepsilon_{\mathrm{e}}$ (extraordinary permittivity) and $\varepsilon^{\prime}{ }_{2}$ $=\varepsilon_{\mathrm{o}}$ (ordinary permittivity).

(ii) $\mathrm{C}_{1}$ and $\mathrm{C}_{2}$ are calculated via Eq. (2).

(iii) $\mathrm{V}(\mathrm{x})$ is obtained by Eq. (6) through the EEC model.

(iv) Voltage distribution, $\mathrm{V}(\mathrm{x})$, determines angular position of the molecules (through Frank-Oseen equations).

(v) A new molecular distribution gives a new effective permittivity distribution in the x-axis, $\varepsilon(\mathrm{x})$. Next, it is averaged in that axis, about the lens diameter, to get a single value. 


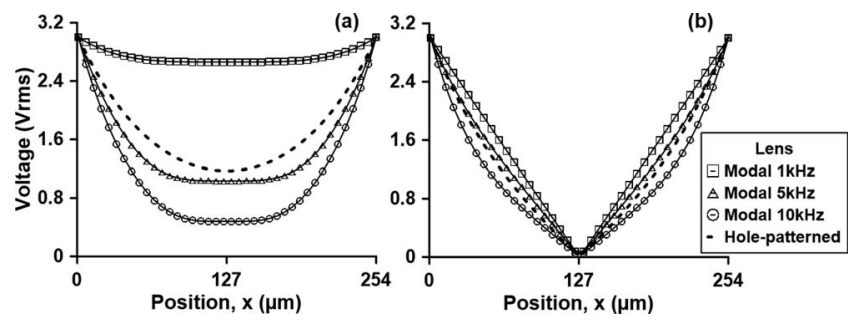

FIG. 3. Voltage distributions across a LC lens diameter for $\mathrm{p}=0.5$. Modal and hole-patterned schemes are plotted. $0^{\circ}$ (a) and $\pi$ (b) electrical phase shifts between electrodes are considered for $3 \mathrm{~V}_{\mathrm{rms}}$.

(vi) Finally, the loop jumps again to the step (i), deducing a new $\mathrm{p}$ value, etc.

This process converges to the final voltage distribution, as there are no changes in the $p$ parameter and the error tolerance is fulfilled.

First, some simulation examples illustrate the voltage across the lens diameter for two LC lenses. The first one has a hole-patterned electrode configuration and the second one governed by modal control. Both lenses have the same constructive parameters (except W1) and are switched to $3 \mathrm{~V}_{\text {rms }}$. The graphs of Fig. 3 correspond to the voltage distribution of case 1 and case 2 , respectively.

Two relevant features are deduced from the results. Frequency dependence of the voltage distribution is noticeable for modal control whereas it is barely relevant, over a decade, for hole-patterned arrangements. This statement is more precisely expressed if it is detailed that the permittivity dispersion has not been considered in this study. Another simulation outcome is a zero crossing voltage that appears at the microlens center, when an inverse driving voltage is applied between electrodes (case 2, Fig. 3(b)) for both schemes and frequency independent. This performance suggests the proposal of a configurable device for a feasible application that switches between lens- and prism-like voltage profiles.

There are no reports about the voltage distribution across a hole-patterned LC lens diameter, to the best of our knowledge. In this work, we have compared the simulated voltage distribution $\mathrm{V}(\mathrm{x})$ from the EEC to the one obtained experimentally. The comparison has been made with a cylindrical hole-patterned array lens manufactured for this purpose. The main array characteristics are: a $254 \mu \mathrm{m} \mathrm{mi-}$ crolens diameter, a $100 \mu \mathrm{m}$ thickness, and it is filled with a MDA 98-1602 nematic LC with a medium birefringence, $\Delta \mathrm{n}=0.2666$. The EEC model predicts the response of the manufactured lens. The results of birefringence and voltage distribution are shown in Fig. 4. On the other hand, a complete characterization of the device has been carried out. Next, there is a rough description of the protocol steps that we have introduced for extracting the experimental voltage distribution, $\mathrm{V}(\mathrm{x})$. The phase retardation of the lens, $\Phi(\mathrm{x})$, has been reconstructed by analysis of the typical interference patterns captured placing the LC lens arrays between crossed polarizers. The unwrapped phase has been demodulated by a skeletonizing technique. In addition, the birefringence, $\Delta \mathrm{n}(\mathrm{x})$, can

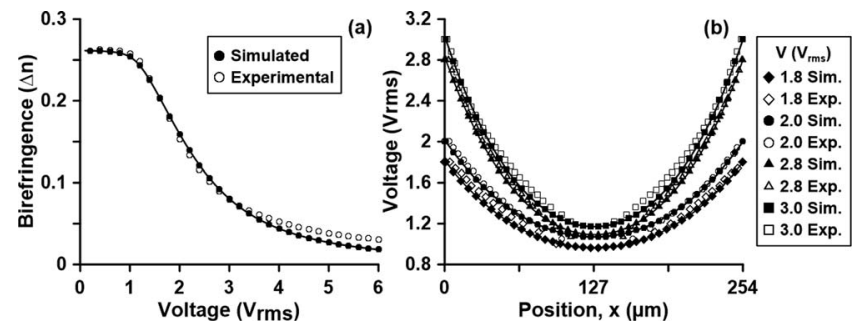

FIG. 4. (a) Simulated and experimental birefringences of a MDA 98-1602 nematic LC. (b) Simulated and experimental voltage distributions when $1 \mathrm{kHz}$ square signal of $\mathrm{V}\left(\mathrm{V}_{\mathrm{rms}}\right)$ is applied to the lens.

be determined as follows:

$$
\Delta n(x)=\frac{\Delta \Phi(x) \cdot \lambda}{2 \pi \cdot d} .
$$

Previously to the final step, the voltage dependence of the birefringence, $\Delta \mathrm{n}$, for the MDA 98-1602 nematic LC, has been measured by means of a spectrometer. This measurement is a correspondence between birefringence and voltage, thus voltage distribution, $\mathrm{V}(\mathrm{x})$, can be achieved at each position, as shown in Fig. 4(b). It is required to do an offset correction in the solution for a fitting result. This adjustment is set by adding each pixel voltage to each final voltage, which is equivalent to convert the relative phase into absolute phase retardation.

The comparison reveals that the profiles of the voltage distributions are fairly in agreement. The deviations can be attributed to the different data resolution from the simulated EEC and the measurements.

In conclusion, this novel EEC predicts the voltage distribution in LC microlenses for both modal and hole-patterned electrode schemes. In the last configuration the fringe field effect turns relevant as the lens aperture decreases. A coplanar capacitor has been chosen as the electric component to model the electric response accurately. An accurate design of some constructive parameters, such as thickness or diameter, can be made previously to the device manufacture. In addition, it could lead to other key estimations of phase profiles, focal lengths, aberrations, etc. The model is also valid for spherical microlenses considering radial symmetry.

The authors are grateful to Grupo de Fotónica Aplicada (Universidad Politécnica de Madrid) for the use of their facilities to manufacture the device. They thank funding support from the Spanish Ministerio de Economia y Competitividad (Grant No. TEC2009-13991-C02-01) and Comunidad de Madrid (Grant No. FACTOTEM S2009/ESP/1781).

${ }^{1}$ S. Sato, Opt. Rev. 6(6), 471 (1999).

${ }^{2}$ P. J. W. Hands, A. K. Kirby, and G. D. Love, Proc. SPIE 5518, 136 (2004).

${ }^{3}$ S. P. Kotova, V. V. Patlan, and S. A. Samagin, Quantum Electron. 41(1), 58 (2011).

${ }^{4}$ M. R. Costa, R. A. C. Altafim, and A. P. Mammana, IEEE Trans. Dielectr. Electr. Insul. 13(1), 204 (2006).

${ }^{5}$ X. Liu, X. Jin, and J.-H. Lee, Solid-State Electron. 53, 1041 (2009).

${ }^{6}$ A. Levy, R. Shavit, and L. Habib, IET Microwaves, Antennas Propag. 4(12), 2133 (2010).

${ }^{7}$ R. Igreja and C. J. Dias, Sens. Actuators, A. 112, 291 (2004).

${ }^{8}$ O. G. Vendik, S. P. Zubko, and M. A. Nikol'skiü, Tech. Phys. 44(4), 349 (1999). 